combined ether extracts were washed with concentrated sodium chloride solution and dried over magnesium sulfate. Evaporation of the ether gave a brownish crystalline mass. Recrystallization from hot water gave thin white plates, mp 119-120 ; yield $40 \%$ ir $1705(\mathrm{C}=\mathrm{O}), 1650$ ( $\mathrm{COOH}$ hydrogen bonded), 3380, 3250, $1395,1190(\mathrm{OH}), 1595,1500,1455$ (aromatic ring) $\mathrm{cm}^{-1}$.

1,4-Dihydroxy-2-(2'-carbethoxyethyl)benzene.-Compound V was converted to the ethyl ester in anhydrous ethanol with hydrogen chloride gas as catalyst. Recrystallization from toluenehexane gave small white needles, $\operatorname{mp~} 78-79^{\circ}$; yield, $78 \%$; ir $3350,1370(\mathrm{OH}), 1610,1525,1450$ (aromatic ring), 1700, 1200 (ester) $\mathrm{cm}^{-1} ; \mathrm{nmr} 3.10(\mathrm{~s}), 3.35(\mathrm{~m}), 4.14(\mathrm{~s}), 5.85$ (qu), 7.25 (m), and $8.78 \mathrm{ppm}(\mathrm{t}) .^{19}$

Oxidation of Hydroquinone Acids and Esters. General Method.-The same general method was used as already reported, ${ }^{2 a}$ using ferric chloride as oxidizing agent in an ethanolwater mixture.

1,4-Benzoquinone-2,5-bis ( $3^{\prime}$-propanoic acid).-Recrystallization from tetrahydrofuran by addition of heptane gave yellow needles, $\mathrm{mp} 191-193^{\circ}$; yield $86 \%$; ir $1710,1228(\mathrm{COOH})$, 1655,1620 (quinone) $\mathrm{cm}^{-1}$.

Anal. Calcd for $\mathrm{C}_{12} \mathrm{H}_{12} \mathrm{O}_{6}$ : C, 57.14; $\mathrm{H}, 4.80$. Found: $\mathrm{C}$ $57.25 ; \mathrm{H}, 4.45$.

1,4-Benzoquinone-2-( $3^{\prime}$-propanoic acid) was obtained as small,

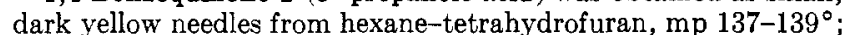
yield $72 \%$; ir $1710,1210(\mathrm{COOH}), 1660,1598$ (quinone) $\mathrm{cm}^{-1}$.

Anal. Calcd for $\mathrm{C}_{9} \mathrm{H}_{8} \mathrm{O}_{4}: \mathrm{C}, 60.25 ; \mathrm{H}, 4.47$. Found: $\mathrm{C}$, $60.35 ; \mathrm{H}, 4.30$.

2-(2'-Carbethoxyethyl)-1,4-benzoquinone was obtained as a bright yellow oil which crystallized on cooling with a Dry Ice-

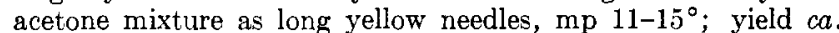
$85 \%$; ir $1730,1253(\mathrm{COOH}), 1660,1600$ (quinone) $\mathrm{cm}^{-1}$; nmr $\tau 3.25(\mathrm{~m}), 5.82(\mathrm{qu}), 7.3(\mathrm{~m})$, and $8.74 \mathrm{ppm}(\mathrm{t}) .^{18}$

$1^{\prime}, 2^{\prime}$-Bis (2,5-dimethoxyphenyl)ethane (VI).-1,4-Dimethoxy-2chloromethylbenzene $[27.7 \mathrm{~g}(0.15 \mathrm{~mol})]$ was dissolved in 120 $\mathrm{ml}$ of tetrahydrofuran. This solution was slowly added to a mixture of $1.82 \mathrm{~g}$ of magnesium turnings and $40 \mathrm{ml}$ of tetrahydrofuran under vigorous stirring, the usual precautions for the preparation of Grignard compounds being taken. After the addition was finished, the reaction mixture was refluxed for $4 \mathrm{hr}$, tetra- hydrofuran was distilled off, and the residue was heated to $100^{\circ}$ for $2 \mathrm{hr}$ on a steam bath. The reaction mixture was then acidified with $6 N$ hydrochloric acid and extracted with ether. Evaporation of the ether gave a slightly yellow oil which soon crystallized. Recrystallization from methanol gave white needles, $\mathrm{mp} 72^{\circ}$; yield $86 \%$; $\mathrm{nmr} \tau 3.29(\mathrm{~s}), 6.20(\mathrm{~s}), 6.28(\mathrm{~s})$, and 7.15 ppm (s).10

$1^{\prime}, 2^{\prime}$-Bis(2,5-dihydroxyphenyl)ethane (I).-Compound VI, 8.0 $\mathrm{g}$, was refluxed for $4 \mathrm{hr}$ with $75 \mathrm{ml}$ of $48 \%$ hydrobromic acid. From the filtered reaction mixture, dark brown crystals separated on cooling. Recrystallization from a large amount of boiling water gave white needles $(4.0 \mathrm{~g})$ which were further purified by sublimation at $200^{\circ}(0.3 \mathrm{~mm})$ bath temperature. They melted at $225^{\circ}$ with decomposition. The same compound was simultaneously prepared by Manecke and Zerpner:13 ir 3210,1375 , $1198(\mathrm{OH}), 1620,1570,1455$ (aromatic ring) $\mathrm{cm}^{-1}$.

$1^{\prime}, 2^{\prime}$-Bis(2,5-benzoquinonyl)ethane (III).-Compound I, $1.2 \mathrm{~g}$, in $50 \mathrm{ml}$ of tetrahydrofuran was oxidized by stirring for $30 \mathrm{~min}$ with $5 \mathrm{~g}$ of silver oxide and $3 \mathrm{~g}$ of magnesium sulfate. The mixture was filtered rapidly, and the solvent was removed in vacuo. The dark brown residue, $1.0 \mathrm{~g}$, was purified by sublimation at $160^{\circ}(0.3 \mathrm{~mm})$ bath temperature to yield microscopic yellow crystals, $\mathrm{mp} 194^{\circ}$; ir 1660,1603 (quinone) $\mathrm{cm}^{-1}$.

Anal. Calcd for $\mathrm{C}_{14} \mathrm{H}_{12} \mathrm{O}_{4}:$ C, $68.85 ; \mathrm{H}, 4.95$. Found: C, $69.18 ; \mathrm{H}, 4.30$.

Registry No.-2'-Hydroxyethyl-2,5-benzoquinone, 4082-30-8; III, 20452-50-0; IV, 20452-51-1; V, 10538-47-3; VI, 20306-76-7; 1,4-dihydroxy-2-(2'-carbethoxyethyl)benzene, 20452-54-4; 1,4-benzoquinone-2(3'-propanoic acid), 20452-56-6; 2-(2'-carbethoxyethyl)-1,4-benzoquinone, 20452-57-7.

Acknowledgment.-We are glad to acknowledge the technical assistance of Mrs. Irmlind Stronkowski in this work, which was supported by a Public Health Service Research Grant GM 10864, National Institutes of Arthritis and Metabolic Diseases.

\title{
Nuclear Magnetic Resonance Spectroscopy. Proton Spectra of Diallylmercury ${ }^{1}$
}

\author{
Herman E. Zieger² ANd John D. Roberts \\ Gates and Crellin Laboratories of Chemistry, California Institute of Technology, Pasadena, California \\ 91109 \\ Received March 6, 1969 \\ Changes have been observed in the nmr spectra of diallylmercury as a function of temperature which seem \\ explicable in terms of allylic rearrangement and intermolecular exchange. Analysis of the proton spectra of \\ diallylmercury spectra using a modified LAOCOON II computer program gave coupling constant and chemical \\ shift parameters which reproduced the spectra at 60,100 , and $220 \mathrm{MHz}$.
}

Analyses of the nmr spectra of three dipropenylmercury compounds have been reported with the objective of establishing their configurations and the stereochemistry of their preparation from propenyllithiums. ${ }^{3}$ Similar studies of di-2-propenylmercury (diallylmercury) do not appear to have been published, ${ }^{4}$ although it offers the additional possibility of undergoing both intermolecular and intramolecular exchange which, in principle at least, are distinguishable by nmr. Diallylmercury is expected to have a carbon-metal bond intermediate in ionic character between tetraallyltin ${ }^{5}$

(1) Supported in part by the National Science Foundation.

(2) On sabbatical leave from Brooklyn College of the City University of New York, 1967-1968.

(3) D. Moy, M. Emerson, and J. P. Oliver, Inorg. Chem., 2, 1261 (1963). (4) However, see references in P. West, J. I. Purmort, and S. V. McKinley. J. Amer. Chem. Soc., 90, 797 (1968), to unpublished work of P. West in which conclusions similar to those of this paper were reached.

(5) D. J. Blears, S. S. Danyluk, and S. Cawley, J. Organometal. Chem., 6 284 (1964). and diallylcadmium, ${ }^{6}$ which have been found to exhibit $A B C D_{2}$ and $A_{4} \mathrm{nmr}$ spectra, respectively. The $\mathrm{AB}_{4}$ spectra observed for allylmagnesium bromide and diallylmagnesium have been interpreted as indicating a rapid allylic rearrangement (either inter- or intramolecular) between the possible allylic isomers. ${ }^{7}$

It was of particular interest to investigate variations in the nmr spectra of diallylmercury with temperature to see whether rearrangement could be detected and whether or not such rearrangement occurs by intermolecular group exchange and can be distinguished from intramolecular rearrangement by disappearance or retention of the ${ }^{199} \mathrm{Hg}$ satellite lines in the progression

(6) K. H. Thiele and J. Kohler, ibid., 7, 365 (1965).

(7) J. E. Nordlander and J. D. Roberts, J. Amer. Chem. Soc., 81, 1769 (1959). G. M. Whitesides, J. E. Nordlander, and J. D. Roberts, ibid., 84, 2010 (1962); Discussions Faraday Soc., 84, 185 (1962). H. E. Zieger and J. D. Roberts, J. Org. Chem., 84, 1976 (1969). 


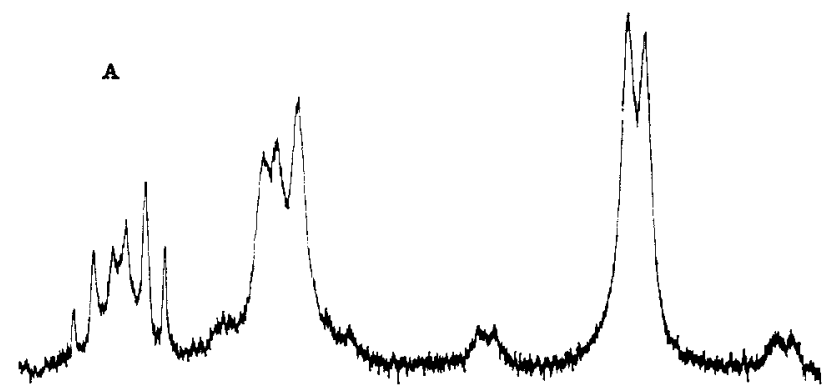

B
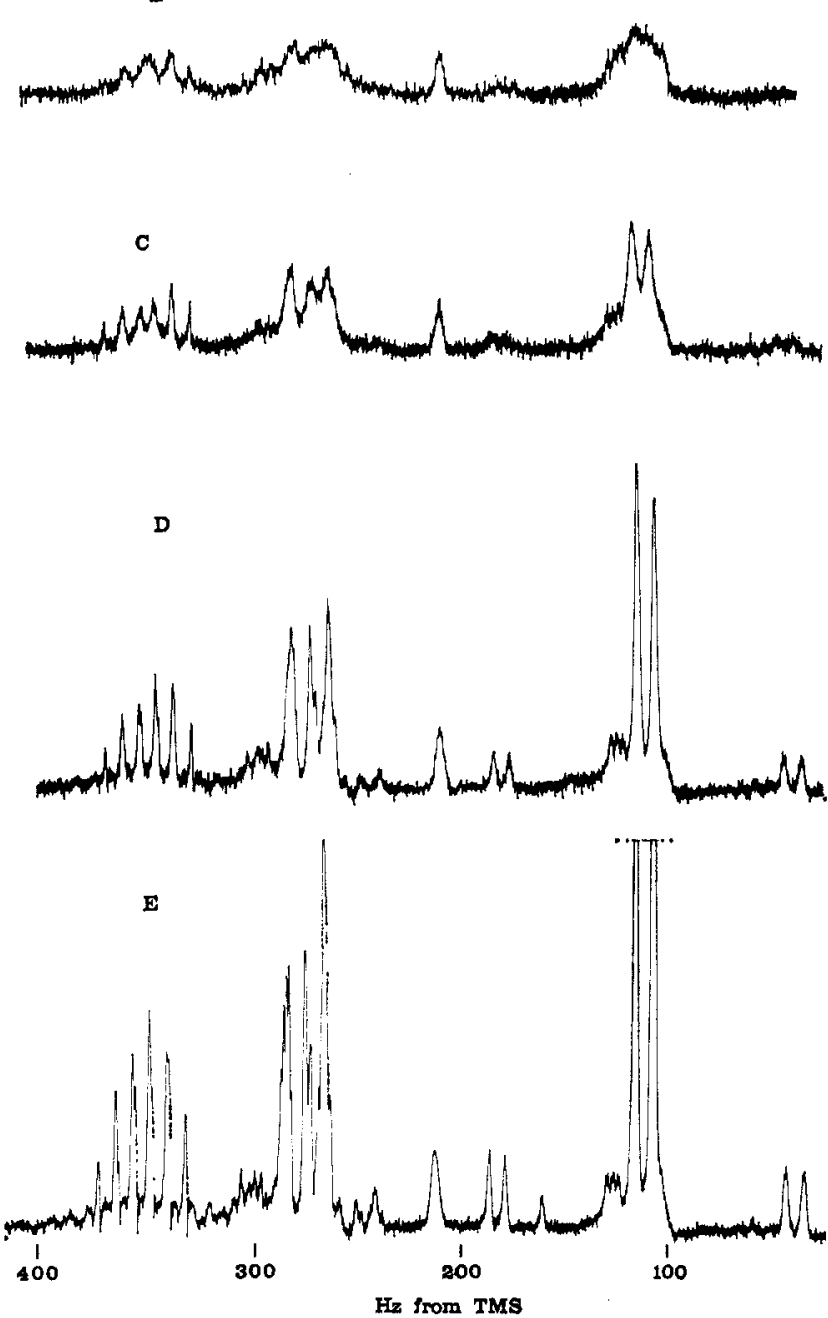

Figure 1.-Proton magnetic resonance spectra of diallylmercury at $60 \mathrm{MHz}$ : $A$, neat at $39^{\circ}$; $B$, in perdeuteriotetrahydrofuran solution at $93^{\circ} ; \mathrm{C}$, at $74^{\circ} ; \mathrm{D}$, at $39^{\circ}$; and $\mathrm{E}$, at $-30^{\circ}$.

of formation of an $\mathrm{AB}_{4}$-type spectrum from an $\mathrm{ABCD}_{2}$ spectrum.

As a prelude to this, analyses were carried out of the $60-\mathrm{MHz}$ and $100-\mathrm{MHz}$ diallylmercury spectra with the aid of a modified LAOCOON II computer program. The coupling constant and chemical shift parameters so obtained were then checked with $220-\mathrm{MHz}$ spectra.

\section{Results}

The $60-\mathrm{MHz}$ spectrum of neat diallylmercury (Figure 1) shows relatively broad, poorly resolved lines which result from a superposition of spectra of the $\mathrm{ABCD}_{2}$ and $\mathrm{ABCD}_{2} \mathrm{X}$ types. ${ }^{8}$ Upon cooling, the spec- tral lines sharpen noticeably. The same behavior results on dissolution in solvents such as tetrahydrofuran (Figure 1) or carbon tetrachloride. A combination of dilution and cooling was found to provide the maximum achievable resolution. Considerable line broadening was observed on heating in tetrahydrofuran (Figure 1), but even at $108^{\circ}$, the highest temperature attempted, the spectra were essentially of the $\mathrm{ABCD}_{2}$ type and it appeared unlikely that $\mathrm{AB}_{4}$ (and possibly $\mathrm{AB}_{4} \mathrm{X}$ ) spectra could be observed at any reasonable temperature for tetrahydrofuran solutions.

These observations, although far from clean-cut, may be interpreted in terms of concomitant allylic rearrangement and intermolecular exchange. The argument for intermolecular exchange is that degradation of the $A B C D_{2} X$ spectrum in the direction of an $A B C D_{2}$ spectrum seems qualitatively faster than the degradation of the $A B C D_{2}$ spectrum in the direction of an $\mathrm{AB}_{4}$ spectra. Exclusive intramolecular rearrangement would be expected to lead to $\mathrm{AB}_{4}$ spectra with mercury satellites and probably would be less dependent on concentration than is in fact observed.

The $n m r$ spectra of diallylmercury were analyzed by essentially conventional techniques. Chemical shifts and coupling constants estimated by first-order approximation from $60-\mathrm{MHz}$ and $100-\mathrm{MHz}$ spectra provided trial spectral-line positions for use in the iterative LAOCOON II program.9,10 The parameters so obtained are given in Table I with the nuclei numbered as in 1. The theoretical spectrum corresponding to these<smiles>C=CCCCC</smiles>

1

parameters gave an acceptable match to the experimental spectrum (Figure 2). ${ }^{11}$ The agreement between the parameters obtained for 60,100 and $220 \mathrm{MHz}$ is quite satisfactory.

The small variations of some of the coupling constants (Table I) observed on cooling diallylmercury in tetrahydrofuran solution could be caused by small changes in the populations of various conformational isomers.

\section{Experimental Section}

Diallylmercury ( $\mathrm{mp}-41$ to $-40^{\circ}$ ) was prepared from allylmagnesium bromide, following the literature. ${ }^{12}$ The sample used for the spectral analysis was dissolved $1: 4(\mathrm{v} / \mathrm{v})$ in perdeuteriotetrahydrofuran and sealed under pre-purified nitrogen in a thick-wall, precision-bore tube. The $60-\mathrm{MHz}_{z}$ spectra were obtained with a Varian Associates A-56/60-A spectrometer equipped with a modified V-6040 variable temperature controller at $39,57,63.5,74$, and $93^{\circ}$, and calibrated with a Hewlett-

(8) Naturally occurring mercury contains $16.9 \%{ }^{190} \mathrm{Hg}$ of spin $I=1 / 2$ which is coupled with the proton spins to give an $A B C D_{2} X$ spectrum.

(9) S. Castellano snd A. A. Bothner-By, J. Chem. Phys, 11, 3863 (1964) (10) A. A. Bothner-By, S. Castellano, and H. Gunther, J. Amer. Chem. Soc., 87, 2439 (1965)

(11) The theoretical spectrum shown in Figure 2 is a computer-produced composite of $83 \%$ of an $A B C D_{2}$ spectrum and $17 \%$ of an $\mathrm{ABCD}_{2} \mathrm{X}$ spectrum (both with the same $\mathrm{ABCD}_{2}$ parameters) from a slightly modified uAocoos II program which permits addition of up to six independent spectra in selected proportions.

(12) A. E. Borisov, I. S. Saveljeva, and S. R. Serdyuk, Bull. Acad. Sci. USSR, Div. Chem. Sci., $896(1965)$; original Russiona appears in Izv. Akad. Nauk SSSR, Ser. Khim., 5, 924 (1965). 


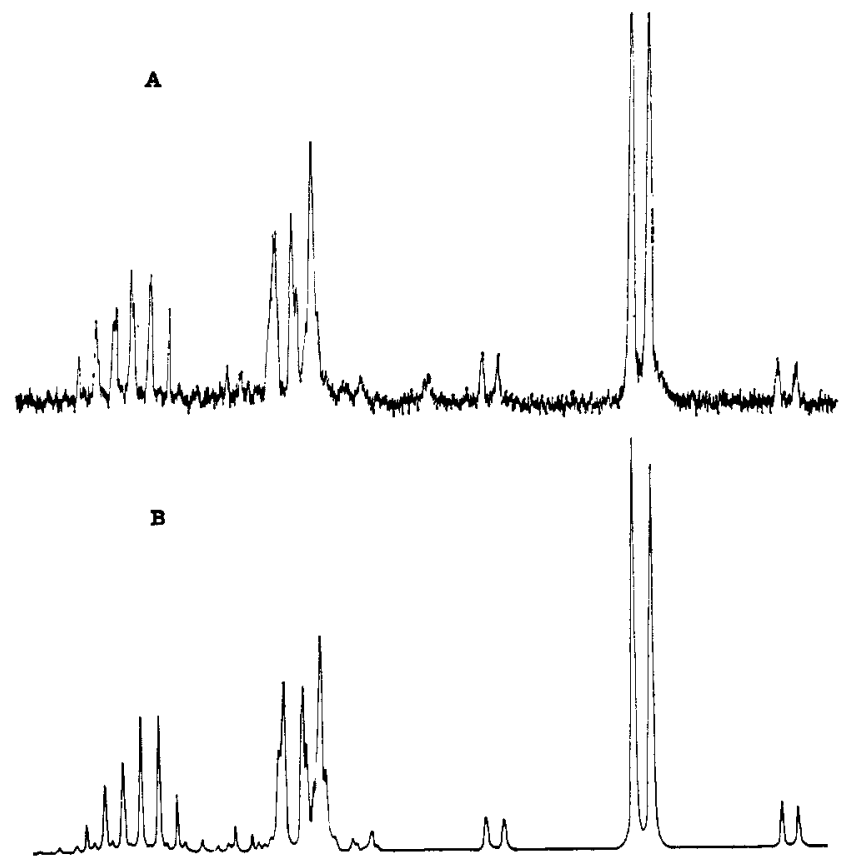

Figure 2.-Experimental proton spectrum (A) of diallylmercury in perdeuteriotetrahydrofuran at $60 \mathrm{MHz}$ and calculated spectrum (B) using the chemical shift and coupling parameters for the $60-\mathrm{MHz}$ analysis as listed in Table I.

Packard 4204A audiooscillator by linear interpolation between TMS side bands. The $100-\mathrm{MHz}$ spectra were obtained on a Varian Associates HA-100 spectrometer operated in the frequency-sweep mode. A Hewlett-Packard V-4315 frequency counter permitted measurement of the line positions to $\pm 0.1 \mathrm{~Hz}$.

Spin decoupling at $100 \mathrm{MHz}$ of nuclei $\mathrm{H}-4$ and $\mathrm{H}-5$ caused simplification of the H-1 multiplet to a symmetrical quartet centered at $-599.3 \mathrm{~Hz}$ from TMS with a total separation of 27.2
TABLE I

Chemical Shifts and Coupling Constants in

Hertz for Diallylmercury (1) in

Perdeuteriotetrahydrofuran

$\begin{array}{lcr} & 100 \mathrm{MHz}^{a} & 60 \mathrm{M} \mathrm{Hz}^{b} \\ \delta_{1} & 598.10 & 360.24 \\ \delta_{2} & 455.38 & 273.52 \\ \delta_{3} & 467.60 & 281.17 \\ \delta_{4}=\delta_{5} & 186.48 & 113.04 \\ J_{12} & 9.52^{c} & 9.43 \\ J_{13} & 16.75^{d} & 17.09 \\ J_{14}=J_{15} & 8.64 & 8.81 \\ J_{23} & 2.21 & 2.21 \\ J_{24}=J_{25} & -0.66 & -0.63 \\ J_{34}=J_{35} & -1.04 & -0.98 \\ J_{16^{e}} & & 45.85 \\ J_{26^{e}} & & 48.82 \\ J_{36^{e}} & & 49.96 \\ J_{46} & & 144.30^{f}\end{array}$

a At $32^{\circ}{ }^{\circ}{ }^{\circ}$ At $\sim 37^{\circ} .{ }^{\circ} 9.5 \mathrm{~Hz}$ from the $220-\mathrm{MHz}$ spectrum. ${ }^{d} 16.6 \mathrm{~Hz}$ from the $220-\mathrm{MHz}$ spectrum. ${ }^{\circ} 8.1 \mathrm{~Hz}$ at $-16.5^{\circ}$ and $8.0 \mathrm{~Hz}$ at $-38^{\circ}$. $f 147.0 \mathrm{~Hz}$ at $-16.5^{\circ}$ and $147.5 \mathrm{~Hz}$ at $-38^{\circ}$.

$\mathrm{Hz}$. The spacing of this quartet suggested that $J_{12}$ was about $10 \mathrm{~Hz}$ and $J_{13}$ about $17.2 \mathrm{~Hz}$, which values were used as original input in the LAOCOON computer program.

Subsequently, a $220-\mathrm{MHz}$ spectrum of diallylmercury obtained on a Varian Associates HR-220 spectrometer gave essentially first-order resonances of $\mathrm{H}-2$ and $\mathrm{H}-3$ which gave $J_{12}$ and $J_{13}$ as 16.6 and $9.5 \mathrm{~Hz}$, respectively.

Registry No.--Diallylmercury, 2097-71-4.

Acknowledgment.-We thank Professor S. I. Chan for assistance in securing $100-\mathrm{MHz}$ spectra and for valuable suggestions. Mr. J. H. Prestegard obtained the $220-\mathrm{MHz}$ spectrum and carried out the spindecoupling experiment.

\title{
Bromine Addition to Olefins in Aqueous Solution
}

\author{
Dipak Acharya and Mihir Nath Das \\ Physical Chemistry Laboratories, Jadavpur University, Calcutta-82, India
}

Received September 12, 1967

\begin{abstract}
Rates of addition of bromine to methyl acrylate, methyl crotonate, methyl methacrylate, and acrylamide in aqueous solutions have been measured electrometrically in the presence of added bromide at 20,30 , and $40^{\circ}$. The rate constants for addition of $\mathrm{Br}_{2}$ as well as of $\mathrm{Br}_{3}$ - have been calculated and hence the corresponding values of activation energy and entropy of activation have been computed for each reaction. The relative reactivities of the four olefins follow the same order (methacrylate $>$ crotonate $>$ acrylamide $>$ acrylate) with respect to both bromine and tribromide ion. For reaction with bromine, activation energy as well as frequency factor lies in the order acrylate $>$ crotonate $>$ acrylamide $>$ methacrylate. For tribromide ion, activation energy lies in the order acrylamide $>$ methacrylate $>$ acrylate $>$ crotonate, the sequence for the frequency factor being acrylamide $>$ methacrylate $>$ crotonate $>$ acrylate. The results are not strictly in conformity with what should be expected from structural considerations.
\end{abstract}

Rates of addition of bromine to several olefins in aqueous solutions were measured by Kanyaev ${ }^{1,2}$ and he concluded that the relative reactivity of molecular bromine and tribromide ion varies continuously with the reactivity of the olefin and also that the proportion of the dibromide in the product is equal to the fraction of reaction effected by tribromide ion. No support for this view has been provided by the studies of Atkinson and Bell. ${ }^{3}$ From their kinetic results, coupled with product analysis, Atkinson and Bell postulated a

(1) N. P. Kanyaev, J. Gen. Chem. USSR, 26, 3037 (1956).

(2) N. P. Kanyaer, ibid. 29, 825 (1959).

(3) J. R. Atkingon and R. P. Bell, J. Chem. Soc., 3260 (1963), general mechanism which is consistent with their findings. More recently Bell and Pring ${ }^{4}$ have reported the kinetic results for addition of bromine to some more olefins in aqueous solution in the presence of added chloride and bromide, which tend to substantiate their earlier conclusions. In general, the velocity constants for reaction with bromine or tribromide ion, varying over eleven powers of ten, show an approximate correlation with the Taft $\sigma^{*}$ substituent constants.

The object of the present investigations was to determine the activation energy and the entropy of activation for the reaction with bromine as well as

(4) R. P. Bell and M. Pring, ibid., B, 1119 (1966), 